\title{
Telessaúde e Covid-19: experiências da enfermagem e psicologia em Foz do Iguaçu
}

\author{
Telehealth and Covid-19: experiences of nursing \\ and psychology in Foz do Iguaçu
}

Mirian Caroline Pereira ${ }^{1}$, Jessica Stranburger da Silva ${ }^{2}$, Tailyse Venialgo Silva ${ }^{3}$, Alessandra Rosa Carrijo ${ }^{4}$, Marcos Augusto Moraes Arcoverde ${ }^{5}$

1. ORCID: orcid.org/0000-0001-5934-2927. Psicóloga, Especialista em Saúde da Família. Universidade Federal da Integração Latino Americana - Unila. Estudante do Mestrado de Saúde Pública em Região de Fronteira. Universidade Estadual do Oeste do Paraná. Foz do Iguaçu, Paraná, Brasil. E-mail: miriancarolinep@gmail.com.

2. ORCID: orcid.org/0000-0003-1336-2337. Graduanda de Enfermagem. Universidade Estadual do Oeste do Paraná, Foz do Iguaçu, Paraná, Brasil. Jeh_stranburger@hotmail.com

3. ORCID: orcid.org/0000-0002-7644-6100. Graduanda de Enfermagem. Universidade Estadual do Oeste do Paraná, Foz do Iguaçu, Paraná, Brasil. tailyse.veni@gmail.com.

4. ORCID: orcid.org/0000-0002-1691-4240. Enfermeira, Doutora em Ciências. Docente. Universidade Estadual do Oeste do Paraná. Foz do Iguaçu, Paraná, Brasil. E-mail: aleenfermeira@msn. com.

5. ORCID: orcid.org/0000-0001-5104-559X. Enfermeiro, Doutor em Enfermagem em Saúde Pública, Docente. Universidade Estadual do Oeste do Paraná. Foz do Iguaçu, Paraná, Brasil. E-mail: marcos.arcoverde2013@gmail.com.

CONTATO: Mirian Caroline Pereira | Endereço: Avenida Araucária, n 7966, Jardim Novo Mundo, CEP:85862-100. Telefone: (45) 99902-0346 | E-mail: miriancarolinep@gmail.com

RESUMO A pandemia do novo coronavírus mostrou a necessidade da utilização de meios tecnológicos no exercício das atividades que envolvem a atenção à saúde. Nesse sentido, pretende-se relatar as atividades desenvolvidas em uma Central de Telessaúde com intervenções de Enfermagem e Psicologia no enfrentamento à Covid-19. Utilizou-se o método de estudo descritivo do tipo relato de experiência, desenvolvido em um projeto de extensão em uma 
universidade estadual localizada no extremo oeste do Paraná (Unioeste), no município de Foz do Iguaçu. Para este relato constam as atividades desenvolvidas entre abril e julho de 2020. Os resultados revelaram que as intervenções realizadas, tais como escuta qualificada, orientações e encaminhamentos, permitiram a manutenção dos cuidados de prevenção e promoção à saúde. Conclui-se que a experiência vivenciada nos possibilitou perceber que é possível prestar atendimentos na área de Enfermagem e Psicologia mediante uso de tecnologia remota, para evitar maiores agravos na saúde da população.

DESCRITORES: Telessaúde. Covid-19. Telenfermagem. Telepsicologia.

\begin{abstract}
The new coronavirus pandemic showed the need to use technological means to carry out activities that involve health care. In this sense, we intend to report the activities developed in a Telehealth Center with Nursing and Psychology interventions to face Covid-19. We used the descriptive study method of the type of experience report, developed in an extension project in a state university located in the extreme west of Paraná (Unioeste), in the city of Foz do Iguaçu. This report includes the activities developed between April and July 2020. The results showed that the interventions performed, such as qualified listening, guidelines and referrals, allowed the maintenance of preventive care and health promotion. It is concluded that the experience made it possible for us to realize that it is possible to provide care in the area of Nursing and Psychology through the use of remote technology, to avoid further damage to the population's health.
\end{abstract}

DESCRIPTORS: Telehealth. Covid-19. Telenursing. Telepsychology.

\title{
INTRODUÇÃO
}

$\mathbf{N}$

- Brasil, o Programa Telessaúde iniciou-se em 2007, para dar apoio à Atenção Básica, e em 2011 o Ministério da Saúde (MS), por meio da Portaria $n^{\circ}$ 2.546, redefiniu e ampliou o Programa Telessaúde Brasil, que passou a ser chamado de Programa Nacional Telessaúde Brasil Redes, com o objetivo de fornecer aos profissionais e trabalhadores das Redes de Atenção à Saúde no SUS os seguintes serviços: Teleconsultoria, Telediagnóstico e Teleducação. O Ministério da Saúde incorporou a Telessaúde como componente da Estratégia e-Saúde (Saúde Digital), com a finalidade de "expansão e melhoria da rede de 
serviços de saúde, sobretudo da Atenção Primária à Saúde (APS), e sua interação com os demais níveis de atenção fortalecendo as Redes de Atenção à Saúde (RAS) do SUS"1.

Cabe destacar que, em outros cenários, o uso da Telessaúde no campo da Enfermagem e da Psicologia já eram praticados em momentos anteriores. A telenfermagem começou a ser desenvolvida no Brasil em 2000, pelo Grupo de Estudos e Pesquisas em Comunicação no Processo de Enfermagem (GEPECOPEN). Por trabalharem em várias vertentes na área de Comunicação em Enfermagem e Saúde, um dos projetos de telenfermagem desenvolvidos consistia na utilização da videoconferência como tecnologia de apoio para demonstração de procedimentos de enfermagem para o paciente, à distância e em tempo real².

A psicologia e os meios digitais também estão coexistindo desde 2000 no Brasil. A telepsicologia como segmento da telessaúde pode utilizar novas tecnologias de informação e comunicação, como telefone, dispositivos móveis, e-mail, chat, mensagens de texto, internet, blogs, sites e videoconferência interativa, na prestação de serviços psicológicos ${ }^{3}$.

Pesquisas em saúde que avaliam a aplicabilidade de meios digitais realizadas com crianças, jovens e adultos com distúrbios de saúde mental demonstram que as intervenções são viáveis, aceitáveis e tão eficazes quanto pessoalmente³.

Pelo exposto, percebe-se que a introdução de ferramentas remotas para auxiliar o cuidado já data de algumas décadas e facilitou o desenvolvimento de novas formas de interação colaborativa entre vários usuários (pacientes e profissionais) com base em mundos virtuais relacionados ao atendimento à saúde logo no início da pandemia da Covid-19. Assim, nesse contexto, a telenfermagem e telepsicologia, bem como a Telessaúde, de modo geral, tornaram-se métodos aceitos e validados para o cuidado e tratamento de muitos problemas de saúde ${ }^{4}$.

No contexto da pandemia da Covid-19, a grande vantagem do uso da tecnologia é oportunizar uma avaliação e orientação de qualidade, evitando que as 
pessoas potencialmente infectadas circulem com o vírus em busca de assistência, sobrecarregando os sistemas de saúde. Embora documentos de referência inicialmente utilizem o termo telemedicina, entendemos que os avanços conquistados permitem ao paciente ter acesso a vários outros profissionais e não apenas ao médico, sendo esse o eixo condutor deste artigo.

Cabe destacar que a Organização Mundial de Saúde (OMS), em 11 de março de 2020, caracterizou como estado de pandemia o surto mundial da doença causada pelo novo coronavírus SARS-CoV-2, também denominada como Covid-19, o que levou os serviços de saúde a um novo cenário de ações em saúde e segurança voltadas aos diversos profissionais envolvidos nos cuidados à população ${ }^{5}$.

Uma das respostas de enfrentamento à pandemia é o isolamento social. Assim, pensando na existência de pessoas que estão em isolamento social e necessitam de assistência à saúde e, para atender a necessidade da população nesse momento de pandemia, o Conselho Federal de Medicina (CFM) encaminhou, no dia 19 de março de 2020, um ofício ao então ministro da saúde, Luiz Henrique Mandetta, informando a decisão de reconhecer a possibilidade de uso da telemedicina no país, decisão que vale em caráter excepcional e enquanto durar o combate à pandemia da Covid-196.

Segundo o documento encaminhado pelo CFM, a telemedicina pode ser exercida nos modelos de teleorientação, telemonitoramento (que possibilita que os pacientes sejam monitorados em parâmetros de saúde e/ou doença) e teleinterconsulta (que permite a troca de informações e opiniões entre médicos, para auxílio diagnóstico ou terapêutico) ${ }^{7}$.

Nesse caminho, percebeu-se que o termo telemedicina se apresenta limitado, frente à sua aplicabilidade nos campos da saúde, tecnologia e informação, por apreciar que as atividades da área da saúde não são de exclusividade médica ${ }^{8}$. Portanto, a terminologia "Telessaúde" traz referências mais amplas e é definida como o uso das tecnologias de informação e comunicação para transferir informações de dados e serviços clínicos, administrativos e educacionais em saúde 3 
e, deste modo, abrange os profissionais de saúde em geral, incluindo enfermeiros, médicos, fisioterapeutas, farmacêuticos, técnicos, psicólogos, entre outros.

Diante da pandemia da COVID-19, o Sistema de Conselhos de Psicologia também vem acompanhando orientações dos órgãos governamentais e atualizando orientações para a categoria de psicólogas(os). Visando evitar o alastramento da pandemia, o Conselho Federal de Psicologia (CFP) possibilitou que psicólogas(os) prestem atendimento à distância (teletrabalho, atendimento online, atendimento telefônico, orientações por e-mail etc.) sem a aprovação prévia no cadastro Nacional de Profissionais de Psicologia para Prestação de Serviços Psicológicos por meio de Tecnologias da Informação e Comunicação (TICS); entretanto, o cadastro é obrigatório e essa medida está prevista durante o período de pandemia do novo coronavírus, conforme Resolução CFP nº 001/2020.

O Conselho Regional de Psicologia do Paraná vem buscando construir materiais e orientações para auxílio da categoria na produção de conteúdo (fundamentados na Psicologia) que auxiliem a população, que passem informações de fontes confiáveis na transmissão do papel social da Psicologia e busquem promover a saúde e a qualidade de vida das pessoas e das coletividades, contribuindo para a promoção do acesso da população às informações, ao conhecimento da ciência psicológica, aos serviços e aos padrões éticos da profissão.

Da mesma forma, o Conselho Federal de Enfermagem (COFEN) autorizou a Teleconsulta de Enfermagem como forma de combate à epidemia provocada pelo novo coronavírus (Sars-Cov-2), considerando a Lei $n^{\circ} 7.498$, de 25 de junho de 1986, que dispõe sobre o exercício da enfermagem, regulamentada pelo Decreto Federal n 94.406/1987 e a Resolução COFEN n 568/2018, que regulamenta o funcionamento dos Consultórios e Clínicas de Enfermagem. Assim, por meio da Resolução n 634/2020, o COFEN reafirma "a importância da participação dos enfermeiros no combate à pandemia mediante consultas, esclarecimentos, encaminhamentos e orientações principalmente nesses momentos de isolamento social", considerando que as pessoas precisam de acesso a informações adequadas e seguras, além da possibilidade de atendimento a suas necessidades 
de saúde sem que necessariamente se desloquem aos serviços de saúde como a única ou primeira opção ${ }^{10}$.

Diante disso, cabe destacar que a Resolução COFEN nº 634/2020 foi construída considerando as diretrizes da Organização Mundial da Saúde (OMS) sobre a pandemia pelo novo coronavírus (Sars-Cov-2) e regulariza a teleconsulta de enfermagem como forma de enfrentamento à Covid-19, respeitando os preceitos éticos e científicos da profissão referentes à integridade das informações resultantes da consulta e da assistência prestada ${ }^{11}$.

A oferta de serviços de Telessaúde pressupõe que quando a distância ou a necessidade do distanciamento social é um fator crítico, esses serviços são prestados por profissionais com o apoio de tecnologias de informação e compartilhamento de dados ${ }^{1}$. Assim, as tecnologias de Telessaúde permitem que os profissionais possam expandir os cuidados para quem precisa, onde quer que estejam. Essa modalidade de serviço também pode ser compreendida como uma estratégia para orientar e monitorar pacientes e populações à luz de suas necessidades. Esses serviços facilitam o acesso, economizando tempo, recursos e promovendo maiores possibilidades de autocuidado ${ }^{10}$, além de que, em um contexto da pandemia, evitam a disseminação da doença.

Assim, o objetivo desse trabalho é relatar as atividades desenvolvidas em uma Central de Atendimento de Telessaúde com intervenções de Enfermagem e Psicologia no enfrentamento à Covid-19.

\section{MÉTODO}

Estudo descritivo do tipo relato de experiência em uma central de atendimento de Telessaúde durante o desenvolvimento do projeto de extensão universitária "Contribuição das ações de extensão da Unioeste no combate à pandemia do novo coronavírus nas regiões Oeste e Sudoeste do Paraná", da Universidade Estadual do Oeste do Paraná (Unioeste). Embora esse projeto tenha ocorrido 
em várias cidades onde a instituição tem campi, este relato de experiência está focado na Central de Atendimento de Telessaúde em Foz do Iguaçu, município que faz fronteira com Argentina e Paraguai. Referente ao período, privilegiou-se apresentar as atividades desenvolvidas entre abril e julho de 2020. Por se tratar de relato de experiência, os resultados são frutos da experiência dos próprios autores durante a execução das atividades a serem descritas.

\section{RESULTADOS}

Por se tratar de um relato de experiência, entendemos que, ao descrever o processo de efetivação do projeto, já estamos mostrando os resultados, visto que o mais pertinente neste momento foi o processo de execução e organização das ações.

Uma das primeiras ações do referido projeto de extensão foi a criação de uma Central de Telessaúde relacionada à Covid-19, instalada no bloco de informática da Unioeste, Campus Foz do Iguaçu. A equipe foi composta por 12 estudantes (dos últimos anos do curso de graduação em enfermagem), três orientadores (uma psicóloga, um enfermeiro e um profissional do serviço social, todos mestrandos) e dois coordenadores (enfermeiros professores da instituição). Essa equipe foi dividida em três turnos, prestando atendimento das 6 às 24 horas; cada turno era executado por pelo menos cinco participantes do projeto, com rodízio de turno da equipe a cada 15 dias.

O formato inicialmente projetado para a central de atendimento foi o tradicional, a partir de uma linha 0800, na qual a população liga, é avaliada pelo estudante ou profissional enfermeiro ou psicólogo, recebe orientações, podendo ser encaminhado durante a mesma ligação para o hospital de referência para Covid-19, caso necessário. Cabe o destaque que, desde o início das ações da central de atendimento, ela esteve ligada à rede de atenção local do Covid-19.

Outra demanda desta central de atendimento foi atender a população me- 
diante a plataforma de Telessaúde Victória Paraná, elaborada pela Secretaria de Estado de Saúde do Paraná - SESA-PR, que esteve em funcionamento de abril a setembro de 2020. O acesso da população a essa plataforma ocorreu por meio do aplicativo "Covid-19 Paraná", disponível gratuitamente na internet. A partir do aplicativo, e mediante uma lista de perguntas básicas pré-estabelecidas, a pessoa informava quais os sinais e sintomas apresentava no momento. Com base nessas informações, a pessoa recebia algumas orientações na tela do celular ou era encaminhada para atendimento com um dos atendentes. O serviço foi organizado para atender toda a população dos 399 municípios do Estado do Paraná. Várias centrais de Telessaúde espalhadas em todas as universidades estaduais do Estado do Paraná foram responsáveis por fazer esse primeiro atendimento. Havendo a necessidade de atendimento médico, o atendente encaminhava o usuário ao profissional.

O aplicativo "Covid-19 Paraná" também disponibilizou à população o atendimento psicológico, devido à demanda de saúde mental de casos apresentados na pandemia ${ }^{12}$. O primeiro atendente, ao realizar avaliação dos sintomas relacionados à Covid-19, ao perceber a necessidade de apoio psicológico com base em perguntas chaves, podia encaminhar o usuário para o acolhimento psicológico que era realizado no mesmo aplicativo. $O$ atendimento psicológico também poderia ser solicitado pelo próprio usuário.

Ao favorecer aos usuários do aplicativo "Covid-19 Paraná" o atendimento remoto de Psicologia, possibilitou-se um sentimento de presença social. Esse sentimento consiste na crença de que as outras pessoas no ambiente virtual são reais e realmente presentes e que o usuário e os demais fazem parte de um grupo e de um processo, mesmo que o contato seja realizado de forma remota. Esse processo foi importante justamente porque foi disponibilizado no momento mais alto do distanciamento social. Assim, o que facilitou o processo de atendimento psicológico, neste contexto, é o fato de as pessoas terem a capacidade de transmitir fortes sentimentos de presença social por meio da interação do aplicativo, aumentando a sensação de união de usuários remotos 
que estão conectados por meio de alguma forma de telecomunicação ${ }^{4}$.

No Brasil, os atendimentos psicológicos seguem modelo de protocolo, o qual traz as normas de funcionamento dos atendimentos no modelo online, seguindo as diretrizes do Conselho Federal de Psicologia (CFP), para segurança e respaldo tanto do profissional como da pessoa que será atendida nesse modelo.

Outra ação desenvolvida pela Central de Telessaúde de Foz do Iguaçu é o atendimento de pessoas que utilizam o aplicativo CovidPR, desenvolvido por professores e alunos do curso de Ciências da Computação da Unioeste, Campus Foz do Iguaçu, também disponível na internet. Este segundo aplicativo, a partir de uma autoavaliação feita pelo usuário, além de fornecer orientações sobre saúde, também orienta sobre os serviços de saúde que estão disponíveis para o enfrentamento da Covid-19 no município.

O CovidPR tem o diferencial de enviar um lembrete diário ao usuário para a sua autoavaliação e fornecer informações oficiais municipais relacionadas à Covid-19 aos usuários. Após a autoavaliação, e conforme os sintomas elencados pelo usuário do aplicativo, a plataforma desse aplicativo emite um alerta destacando os casos classificados como suspeitos e alto suspeitos. A equipe da Central de Telessaúde da Unioeste de Foz do Iguaçu tem acesso a esse alerta de casos (alertas de suspeitos e alto suspeitos). A partir desse momento, a equipe faz o contato com os usuários indicados com alerta, refazendo a avaliação dos sintomas, com o olhar profissional, mediante o protocolo e o fluxo estabelecidos pela Secretaria Municipal de Saúde de Foz do Iguaçu. Com base nessas informações a pessoa recebe orientações ou ainda pode ser encaminhada para dar continuidade ao atendimento já pela equipe do hospital de referência, seguindo o mesmo fluxo de atendimento do 0800.

Todo esse processo possibilita que a equipe da Central de Telessaúde desenvolva um trabalho proativo e de escuta qualificada, a partir das informações da plataforma do aplicativo CovidPR. Cabe destacar que esse aplicativo também tem sido utilizado no monitoramento dos casos que continuam em acompa- 
nhamento. Os usuários são orientados (tanto pela equipe da central de informação, quanto pela equipe hospitalar e da atenção básica) a continuar realizando a autoavaliação diária, assim a equipe que monitora (equipe hospitalar ou da atenção básica) pode ir acompanhando a evolução dos sinais e sintomas, fazendo contato quando necessário.

A Figura 1 apresenta o fluxo de atendimento da Central de Atendimento de Telessaúde da Unioeste, Campus Foz do Iguaçu, e como a mesma se insere na rede municipal de enfrentamento ao Covid-19.

Figura 1. Fluxo de atendimento da Central de Atendimento de Telessaúde no enfrentamento da Covid-19, Universidade Estadual do Oeste do Paraná, Campus de Foz do Iguaçu, 2020.

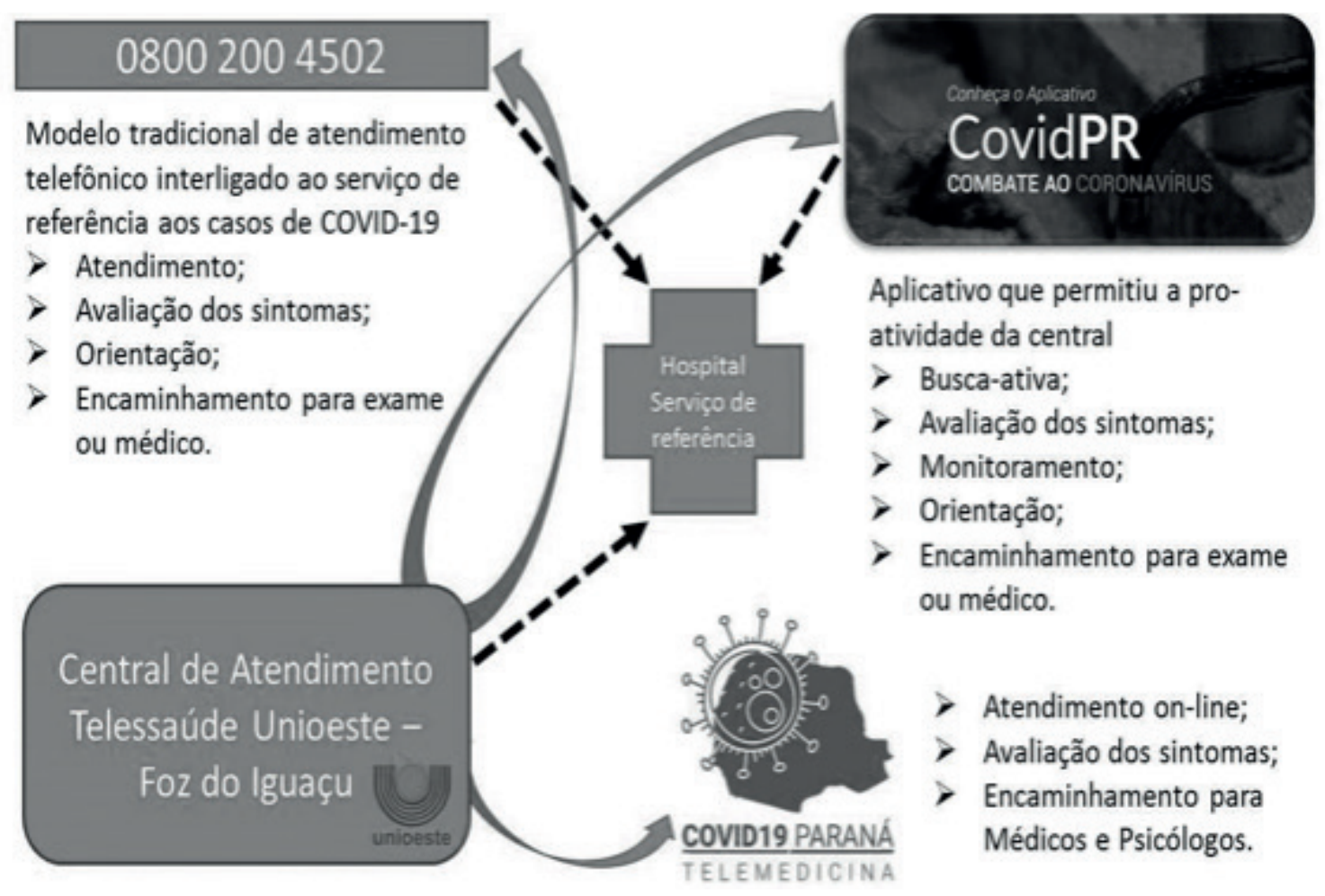

Fonte: Elaborado pelos autores (2020).

\section{DISCUSSÃO}

Com a chegada da Covid-19 no Estado do Paraná, em especial no município de Foz do Iguaçu, frentes de trabalho começaram a se organizar buscando di- 
minuir a disseminação do vírus e os seus impactos, sendo que uma dessas frentes foi o projeto de extensão já mencionado, ao qual está vinculada a Central de Atendimento da Unioeste, Campus Foz do Iguaçu.

A Central de Atendimento, como uma ação de enfrentamento à Covid-19 na modalidade de Telessaúde, mostra-se como estratégia de intervenção para diversos profissionais de saúde ao permitir orientações ao paciente via assistência remota, oferecendo suporte diante das necessidades de saúde, além do encaminhamento para outros serviços, quando necessário. A Telessaúde tem sido utilizada pelos profissionais para identificação de sinais e sintomas de Covid-19 na população e orientações de cuidados referentes à pandemia, realizando até mesmo as consultas, conforme as deliberações dos diversos conselhos profissionais citados neste trabalho. Ações como esta são importantes neste momento de pandemia devido ao alto grau de transmissibilidade do vírus ${ }^{13}$.

Nesse sentido, as orientações fornecidas por telefone fazem parte das estratégias de promoção de saúde, permitem a melhoria do conhecimento e autocuidado e, na situação da pandemia, complementam o serviço de vigilância em saúde e de atenção hospitalar, aperfeiçoando o acesso às informações, o que potencializa as medidas de enfrentamento à Covid-19.

No contexto da telenfermagem, uma autora defende que este recurso, ao ser aplicado em uma situação de crise, pode beneficiar até mesmo casos não urgentes, bem como consegue trazer para os serviços os casos que de fato necessitam de tal assistência ${ }^{14}$.

Ainda sobre esse contexto, uma revisão sistemática se propôs a estudar a telenfermagem em situações de desastres. Chegou-se à conclusão de que o cuidado à saúde mediado por uso da telenfermagem é essencial e oferece um meio de melhorar a resposta à necessidade do outro, mas também identificou escassez de enfermeiras especializadas nesta área de atuação. Desse modo, se faz necessário ter esse profissional altamente capacitado nos programas de telessaúde, sendo que isso abrirá novos horizontes para a profissão ${ }^{15}$. 
Especificamente para a Psicologia, um artigo recente conclui que telepsicologia é crucial para a prestação de serviços psicológicos, mais do que nunca durante a pandemia de Covid-19, sendo pouco provável que essa demanda mude à medida que psicólogos e pacientes passam a dar importância a essa modalidade de atendimento ${ }^{16}$.

A influência da tecnologia tem facilitado a prática de diversos profissionais da saúde, ainda mais neste momento de pandemia ${ }^{10}$; entretanto, a modalidade de assistência não presencial exige treinamento, principalmente no que se refere à comunicação interpessoal. Percebe-se que uma vez que a tecnologia tem sido incorporada de maneira substancial na assistência, devem ser criadas estratégias tanto na esfera pública quanto privada que direcionem a atenção nesse quesito ${ }^{17}$. Para que essas dificuldades possam ser superadas, a comunicação é uma competência que deve ser ensinada aos profissionais que atuam na telessaúde, pois, ainda que a tecnologia facilite a prática profissional, é mais difícil se comunicar virtualmente, sobretudo pela dificuldade de percepção de sinais não verbais, obstáculo enfrentado com a adequada capacitação de profissionais quanto ao processo de comunicação interpessoal adequada ${ }^{17}$.

Nesse sentido, alguns autores defendem que a aplicação da telessaúde pode ser desafiadora para os profissionais da saúde, pois, em decorrência do aumento do uso da tecnologia na saúde, os conceitos de cuidado e de interação podem mudar e a fala virtual pode se tornar mais dominante do que as interações pessoais, em algum momento futuro. Deste modo, os componentes essenciais do cuidado devem ser mais discutidos nos serviços de saúde e durante a formação destes profissionais ${ }^{18}$.

Conforme o relato exposto e a literatura citada, as intervenções de telessaúde permitiram o acompanhamento dos pacientes, por intermédio de teleatendimento, momento quando ocorreu a realização de orientações e encaminhamentos importantes para a manutenção dos cuidados de prevenção e promoção à saúde para o enfrentamento da Covid-19. 
Referente ao relato de experiência, como tal, os benefícios elencados neste trabalho não podem ser generalizados a outras realidades, mas podem servir de modelo para novas experiências, principalmente no cenário brasileiro.

\section{CONCLUSÃO}

Os resultados deste relato contribuem para a percepção de que a modalidade de assistência não presencial está se tornando uma ferramenta de apoio significativa para os profissionais da saúde desempenharem algumas funções de forma ágil, devido à sua aplicabilidade no cotidiano. Pode-se pensar que esse modelo de atendimento irá se manter nos serviços de saúde, bem como em outros serviços, tanto na esfera pública quanto privada, devido aos benefícios percebidos ao longo dos atendimentos e acompanhamentos dos casos.

Nesse sentido, a experiência vivenciada na Central de Atendimento em Telessaúde permitiu perceber como profissionais da Enfermagem e Psicologia podem prestar atendimento às pessoas mediante o uso de tecnologia remota, através do apoio da tecnologia incorporada de maneira rápida e ágil na assistência à saúde, o que também permite tornar profissionais mais capacitados para atuação em casos de calamidade pública.

Esse relato, em alguma medida, também demonstrou como os serviços de saúde se organizaram para atender a população de modo remoto como resposta ao enfrentamento da Covid-19 no município de Foz do Iguaçu.

\section{AGRADECIMENTO}

Agradecemos à Fundação Araucária pelo apoio financeiro no fomento das bolsas. 


\section{REFERÊNCIAS}

1. Conselho Regional de Enfermagem de São Paulo. Parecer COREN-SP 038/2019, 2 de dezembro de 2019. Realização de Telenfermagem pelos profissionais de enfermagem. São Paulo: Diário Oficial da União; 2019.

2. Prado C, Silva IA, Soares AVN, Aragaki IMM, Shimoda GT, Zaniboni VF et al. Teleamamentação no Programa Nacional de Telessaúde no Brasil: a experiência da Telenfermagem. USP [Internet]. São Paulo: Rev. esc. enferm.; 2013. [citado em 2020 Set 29]; 47(4):990-996.Disponível em: https://doi.org/10.1590/S0080-623420130000400031.

3. Gorini A, Gaggioli A., Vigna C, Riva G. A second life for eHealth: prospects for the use of 3-D virtual worlds in clinical psychology. Journal of medical Internet research [Internet]. 2008. [citado em 2020 Set 29]; 10(3), e21. Disponível em: https://doi.org/10.2196/ jmir.1029.

4. Reay RE, Looi JC, Keightley P. Telehealth mental health services during COVID-19: summary of evidence and clinical practice [Internet]. Australas Psychiatry; 2020 Jul. [citado em 2020 Set 29]. 28:1039856220943032. Disponível em: https://journals. sagepub.com/doi/full/10.1177/1039856220943032

5. Ministério da Saúde. Folha informativa - COVID-19 (doença causada pelo novo coronavírus). OPAS Brasil; 2020.

6. Piropo TGN, Amaral HOS. Telessaúde, contextos e implicações no cenário baiano. Saúde em debate [Internet]. 2015. [citado em 2020 Jul 10]; 39(104): 279-287. Disponível em: https://doi.org/10.1590/0103-110420151040413.

7. Brasil Telemedicina. Telemedicina é liberada para apoio ao combate do Coronavírus [internet]. São Paulo; 2020. [citado em 2020 Abr 3]. Disponível em: https://brasiltelemedicina.com.br/noticia/telemedicina-liberada-para-apoio-ao-combate-do-coronavirus/.

8. Conselho Regional de Psicologia do Paraná. Nota Técnica CRP-PR nº01/2020, de 20 de março de 2020. Orienta a(o) Psicóloga(o) sobre o atendimento psicológico nas políticas públicas e instituições privadas, diante da pandemia do COVID-19. Diário Oficial da União; 2020.

9. Conselho Regional de Psicologia do Paraná. Nota Técnica CRP-PR nº01/2020, de 20 de março de 2020. Orienta a(o) Psicóloga(o) sobre o atendimento psicológico nas políticas públicas e instituições privadas, diante da pandemia do COVID-19. Diário Oficial da União; 2020.

10. International Council of Nurses (ICN) (2007). International Competencies for Telenursing. Geneva: International Council of Nurses; 2007

11. Almeida BI, Silva MJP. Cuidado de enfermagem por telessaúde: qual a influência da distância na comunicação? [Internet]. Rev. Bras. Enferm.; 2017 Oct. [citado em 2020 Ago 12]; 70(5): 928-934. Disponível em: https://doi.org/10.1590/0034-7167-2016-0142.

12. Faro A, Bahiano MD, Nakano TD, Reis C, Silva BF, Vitti LS. COVID-19 e saúde mental: a emergência do cuidado [Internet]. Campinas: Estudos de Psicologia; 2020. [citado em 2020 Set 29]; 37. Disponível em: https://doi.org/10.1590/1982-0275202037e200074.

13. Caetano R, Silva AB, Guedes ACCM, Paiva CCN, Ribeiro GR, Santos DL et al. Desafios e oportunidades para telessaúde em tempos da pandemia pela COVID-19: uma reflexão sobre os espaços e iniciativas no contexto brasileiro [Internet]. Cad. Saúde Pública; 2020. [citado em 2020 Out 2]; 36 (5): e00088920. Disponível em: https://doi.org/10.1590/0102-311×00088920.

14. Castner J. Special Disaster Issue. Journal of Emergency Nursing; 2020 Set. [citado em 2020 Out 2]; 46(5):560. Disponível em: https://doi.org/10.1016/j.jen.2020.06.012.

15. Nejadshafiee M, Bahaadinbeigy K, Kazemi M, Nekoei-Moghadam M. Telenursing in incidents and disasters: a systematic review of the literature [Internet]. Journal of emergency nursing; 2020 Abr. [citado em 2020 Out 2] 29. Disponível em: https://doi. org/10.1016/j.jen.2020.03.005.

16. Perrin PB, Rybarczyk BD, Pierce BS, Jones HA, Shaffer C, Islam L. Rapid telepsychology deployment during the COVID-19 pandemic: A special issue commentary and lessons from primary care psychology training [Internet]. Journal of Clinical Psychology; 2020 Jun. [citado em 2020 Out 2]; 76(6):1173-85. Disponível em: https://doi.org/10.1002/jclp.22969.

17. Barbosa IA, Silva M J P. Assistência de enfermagem em telessaúde: qual a influência da distância na comunicação? [Internet] Rev. Bras. Enferm.; 2017. [citado em 2020 Set 30]; 70 (5): 928-934. Disponível em: https://doi.org/10.1590/0034-7167-2016-0142.

18. Gunawan J, Aungsuroch Y, Marzilli C. 'New Normal'in Covid-19 Era: A Nursing Perspective From Thailand [Internet]. Journal of the American Medical Directors Association; 2020 Jul; [citado em 2020 Out 2] 21(10): 1514-1515. Disponível em: https://doi. org/10.1016/j.jamda.2020.07.021.

\section{RECEBIDO:03/08/2020 \\ ACEITO:13/10/2020}

